\title{
A Review on Human Body Communication: Signal Propagation Model, Communication Performance, and Experimental Issues
}

\author{
Jian Feng Zhao, ${ }^{1}$ Xi Mei Chen, ${ }^{2}$ Bo Dong Liang, ${ }^{1}$ and Qiu Xia Chen' \\ ${ }^{1}$ The School of Automotive \& Transportation Engineering, Shenzhen Polytechnic, Shenzhen, China \\ ${ }^{2}$ Baoan Urban Renewal Bureau, Shenzhen, China \\ Correspondence should be addressed to Jian Feng Zhao; zjf@szpt.edu.cn
}

Received 22 April 2017; Accepted 11 September 2017; Published 22 October 2017

Academic Editor: David Naranjo

Copyright (c) 2017 Jian Feng Zhao et al. This is an open access article distributed under the Creative Commons Attribution License, which permits unrestricted use, distribution, and reproduction in any medium, provided the original work is properly cited.

\begin{abstract}
Human body communication (HBC), which uses the human body tissue as the transmission medium to transmit health informatics, serves as a promising physical layer solution for the body area network (BAN). The human centric nature of HBC offers an innovative method to transfer the healthcare data, whose transmission requires low interference and reliable data link. Therefore, the deployment of HBC system obtaining good communication performance is required. In this regard, a tutorial review on the important issues related to $\mathrm{HBC}$ data transmission such as signal propagation model, channel characteristics, communication performance, and experimental considerations is conducted. In this work, the development of HBC and its first attempts are firstly reviewed. Then a survey on the signal propagation models is introduced. Based on these models, the channel characteristics are summarized; the communication performance and selection of transmission parameters are also investigated. Moreover, the experimental issues, such as electrodes and grounding strategies, are also discussed. Finally, the recommended future studies are provided.
\end{abstract}

\section{Introduction}

The prevalence of chronic diseases (e.g., heart diseases, diabetics, and strokes) and escalating of aging population have become public health concerns and challenges of healthcare system. According to world health organization (WHO), millions of people suffer from chronic diseases and die, for instance, in 2012, cardiovascular diseases led to 17.5 million deaths and diabetes caused 1.5 million deaths [1]. Moreover, the absolute number of people aged 60 years and over will increase from 900 million to 2 billion from year 2015 to 2050 [2], and aging population is fueling the prevalence of chronic disease as elder people are more prone to have chronic diseases [3]. To effectively control the chronic diseases and improve the quality of life for patients, continuous health monitoring [4] is required. For high risk group people, the continuous monitoring of physiological data helps to early detect and prevent the diseases [5]. For instance, brain and muscles activities need to be continuously monitored in patients with Parkinson's disease and blood glucose is monitored in patients with diabetes. The promising solution to enable the continuous physiological data monitoring is to deploy the body area network (BAN) [6, 7], where tiny-size sensors and actuators are utilized to monitor the physiological data and provide the therapeutic functions (i.e., administrate the insulin dosage, control the rates of cardiac pacemaker, etc.) $[6,8,9]$.

The structure of BAN is shown in Figure 1. The sensor nodes including both the on-body and in-body nodes (implantable devices), generally perform the monitoring function (pulse oximeter measures $\mathrm{SpO}$; blood pressure sensor measures blood pressure). The physiological data from these nodes over a period of time are delivered privately and reliably to a relay node or aggregator mounted on the body, such as a smart-watch or smart wristband $[10,11]$, which are emerging devices in the biomedical industry due to their convenience to control and constantly wear behavior. The data are then forwarded to the hub and the central control point, from where the data are available to hospital, professional staff, and emergency center or for personal usage. 


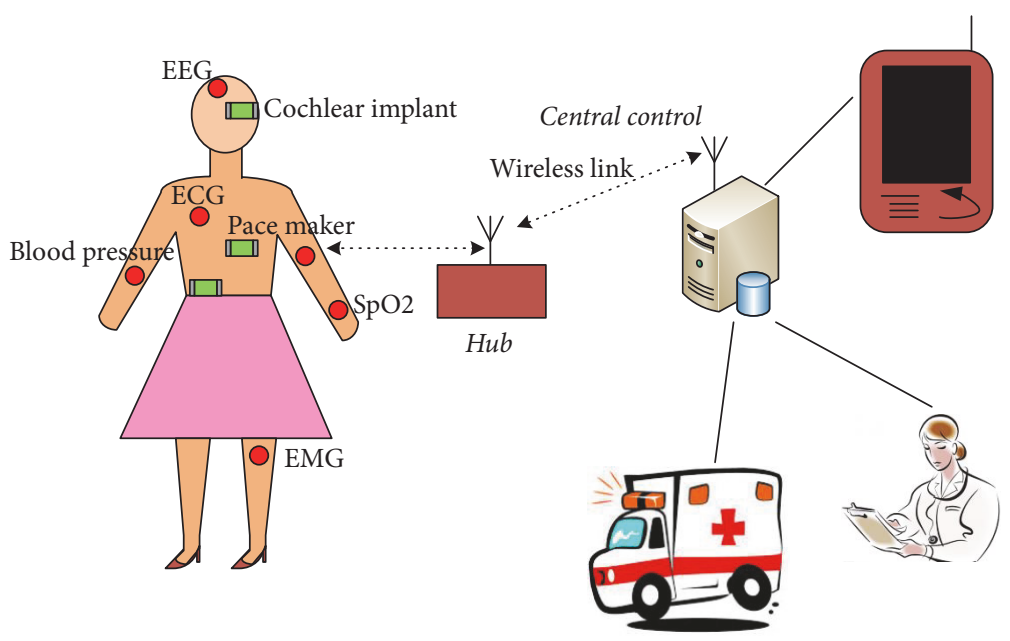

FIGURE 1: Structure of body area network.

Human centric is the major characteristic for BAN, the communication among sensors scattered on/in the human body and aggregator should be accomplished by wired connection or short distance wireless communication techniques. Obviously, wired connection is not a good choice since wire can break and is inconvenient for patients' movement. The short range radio frequency (RF) wireless techniques such as Zigbee, Bluetooth, and Ultrawide Band (UWB) are the preferable candidates. However, these approaches have the intrinsic drawbacks since they are not designed for human centric health monitoring data transmission. For instance, the RF techniques are prone to electromagnetic interference and suffer from large signal leakage and easy eavesdropping. Furthermore, human body is primarily (65\%) composed of water, which is a blocking material for RF signals [12]. Thus the RF wireless signals transmitted around or in the human body will suffer from body-shadowing effect, which will lead to significant signal attenuation $[13,14]$. Moreover, the RF techniques radiate the signal via antenna, which is challenged for miniaturization. Another potential candidate is the inductive wireless coupling technique. However, its coupling efficiency $(0.4 \%$ with $3 \mathrm{~cm}$ distance [15]) is low and coil size (typical dimensions of $25 \mathrm{~mm} \times$ $10 \mathrm{~mm}$ for rectangular coil, diameter $20 \mathrm{~mm}$ for circular coil) [16] is large. Also the coil is a problem for miniaturization. It is evident that new communication techniques targeted for human centric healthcare monitoring data transfer are required for the medical BAN.

Human body communication (HBC, also termed intrabody communication) is a novel transmission technique using the human body as the transmission medium for electrical signal transfer [17]. HBC has become one of three physical layers (another two are narrow band and UWB) for BANs proposed by IEEE 802.15.6 task group 6 [18]. HBC can be implemented in two ways: capacitive coupling method and galvanic coupling method. It has the following desirable characteristics. (a) Little Signal Leakage-High Security. The signal is confined to the surface of the body and with little energy radiated into the surrounding environment (avoid the body acting as antenna to radiate energy) $[19,20]$. Therefore, the security of information is guaranteed for its avoidance of eavesdropping, and the interference among different individuals can be minimized as well.

(b) Low Signal Attenuation-Low Transmission Power. Compared with air channel, human body channel obtains high channel gain [21], which can lower the transmission power. And thus it may potentially lower the power consumption of the system and helpful for miniaturization.

(c) Low Carrier Frequency. HBC operates in low frequency (especially for galvanic coupling $\mathrm{HBC}$ ), which can potentially simplify the design, minimize the power consumption (in HBC system, the detected circuit, filter, and power amplifier have the component of CMOS circuit, in which the dynamic power is proportional to the carrier frequency. Also power consumption of CPU and frequency synthesizer is proportional to operation frequency) $[22,23]$, and reduce the size of devices due to the low requirement on amplifiers, capacitors, and inductors. Meanwhile, the lower carrier frequency requires low-intermediate-frequency receiver, which can potentially achieve the crystal-less receivers due to its better tolerance to the frequency deviation [24]. The crystalless transceiver could further reduce the size and power consumption, since crystal oscillator is bulky, power-hungry, and fragile components. In addition, HBC does not require the antenna, which can minimize the size of chip.

The comparisons of $\mathrm{HBC}$ and other wireless techniques are shown in Table 1. To serve as a suitable communication technique for BAN, $\mathrm{HBC}$ has been attempting to become a prospective candidate due to its low interference, high security (signal intercept requires body touch), suitable transmission range, and potentially higher degree of miniaturization. 
TABLE 1: Comparison of wireless communication techniques.

\begin{tabular}{lcccr}
\hline Technique & $\begin{array}{c}\text { Frequency } \\
(\mathrm{GHz})\end{array}$ & $\begin{array}{c}\text { Data rate } \\
(\mathrm{Mbps})\end{array}$ & $\begin{array}{c}\text { Power } \\
(\mathrm{dBm})\end{array}$ & Distance \\
\hline Zebee [93] & $2.4,0.865$ & 0.25 & $-3 \sim 10$ & $10 \mathrm{~m}$ \\
Bluetooth [94] & 2.4 & $0.721 \sim 24$ & $<20$ & $10 \mathrm{~m}$ \\
UWB & $3 \sim 10$ & $0.85 \sim 20$ & -41 & $10 \mathrm{~m}$ \\
ICL & $0.001 \sim 0.4[15]$ & $0.0001-4[95]$ & - & $0.5 \sim 3 \mathrm{~cm}$ \\
Cap-HBC & $0.01 \sim 0.1$ & $0.002 \sim 10$ & $-15 \sim 5$ & $5 \mathrm{~cm} \sim 2 \mathrm{~m}$ \\
Gal-HBC & $0.0001 \sim 0.01$ & $0.0009 \sim 2$ & $-30 \sim-4$ & high \\
\hline
\end{tabular}

Inf: interference; ICL: inductive coupling link; Cap-HBC: capacitive coupling HBC; Gal-HBC: galvanic coupling HBC.

By using HBC for data transfer among the wearable or implantable healthcare monitoring devices, the low power transmission is important, not only for avoiding information leakage or interference to other BANs, but also for saving power (the decrease of transmission power can lower the power consumption of power amplifier, which accounts for a significant portion of the HBC system power consumption). This is especially true for implantable devices, as the leakage of important information (e.g., pacing speed and pacing threshold setting of pacemaker) to unintended receiver will lead to life-threatening events. In addition, low transmission power is of importance as it can also extend the lifetime of battery [25], which requires costly and invasive surgical operation for replacement.

To achieve low transmission power, it is necessary to minimize transmission power and optimize its communication performance. Certainly, the reliable data transmission should be at the same time maintained. Then the transmission parameters correlated with the transmission power, such as data rate, modulation method, bandwidth, and frequency band, should be selected suitably. That motivates us to summarize the channel characteristics and find out the rule of communication performance (i.e., bit error rate BER) for different modulation schemes. Of course, the experimental issues to obtain the channel characteristic are also of significant. Therefore, having a survey on the above issues is of importance for the deployment of HBC. In what follows, the history and development of $\mathrm{HBC}$ are reviewed. Then the signal propagation model is summarized in Section 3; based on the models, the channel characteristics and communication performance are provided in Section 4. The experimental issues are discussed in Section 5. Finally, the conclusions and future studies are addressed in Section 6.

\section{The Development of Human Body Communication}

2.1. The History of Electrical Property and Human Tissue. In the history of human beings, the interests on body tissues were inevitable. Many researchers devoted their efforts and thoughts to investigate the muscles and their electrical properties and biomechanics. There are generally two forms of bioelectrical assessment of muscle, one is to investigate the electrical signal originated in muscle and another is the properties of muscle by applying electrical signals.
The relationship between electricity and muscle contraction was first observed by the Italian physician Luigi Galvanic in the mid-1780s. Luigi Galvanic performed the experiment of connecting the nerves of recently dead fog to a long metal wire and pointed it towards the sky during thunderstorm, the frog's legs twitched and jumped as if they were alive with flash of lighting, from which Galvanic pointed out that recently dead muscle tissue can respond to external electrical stimuli. Since then, more and more researchers investigated the response of human tissue to electric current, the tissue impedance, and dielectric properties. For example, the nerve, muscle, and gland can be stimulated to produce anesthesis [26] by milliampere electric currents. The early extensive literature reviews on dielectric properties have been provided by Geddes and Baker [27], who summarized the early reports on the specific resistance of tissues. Later, the intensive research on tissue dielectric property was reported by Gabriel et al. [28]. And further experiments were conducted by Gabriel et al. to study the dielectric properties of human and animal tissue in the frequency range $10 \mathrm{~Hz}$ to $20 \mathrm{GHz}$ [29]. Based on these measurements, a parametric model with four Cole-Cole type dispersions was developed to describe the dielectric properties of tissue as a function of frequency [30]. These electrical properties have been utilized by the researchers to facilitate the recent research and applications. For instance, electrical impedance tomography (EIT) has been developed to image the internal organs and structure of body for medical diagnosis; electrical stimulation has been adopted for medical therapy and prosthesis. Until 1995, human body as a transmission medium was proposed to be utilized for data transmission. This type of telemetry, called capacitive coupling HBC [17], was developed to enable data transmission on or around the human body.

The pioneer researches of HBC focused on the feasibility of implementing HBC. After that, the signal propagation models were emerging to investigate the signal propagation mechanism and channel characteristics. Later, experiments and prototypes were developed to investigate their communication performance. Generally, HBC can be implemented in two ways: capacitive coupling method and galvanic coupling method. In what follows, the details will be presented.

\subsection{HBC Coupling Methods and Their First Attempts}

2.2.1. Capacitive Coupling HBC. In 1995, Zimmerman [17] did the research to perform the position measurement of 


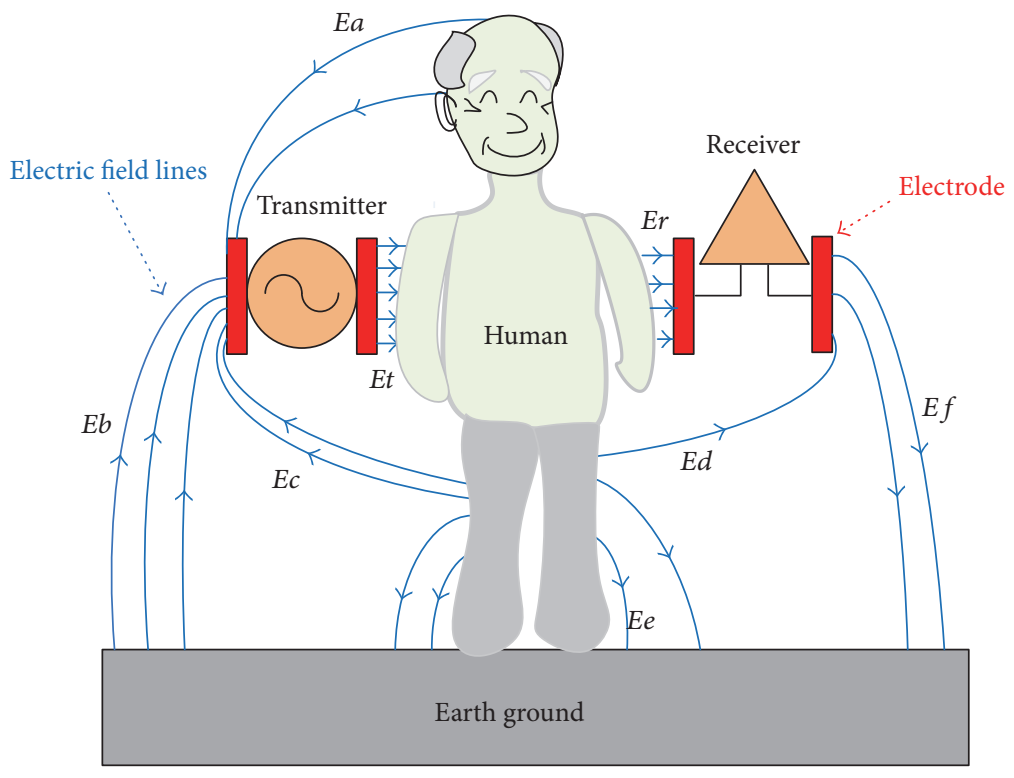

FIgURE 2: Principle of capacitive coupling HBC.

Yo-Yo Ma's cello bow. It was discovered that placing one's hand in electric field attenuates the received signal significantly. Based on these facts and principle, he proposed the concept of Personal Area Network (PAN), a wireless system that allows electronic devices on and near the human body to exchange digital information through $\mathrm{HBC}$, which does not need the sophisticated antenna to radiate the signal into air; instead, electrodes are used to couple the electric signal to human body, which is then served as the medium to convey the signal. Zimmerman also developed a prototype as an initial attempt to implement HBC technology. This prototype validates the feasibility of using $\mathrm{HBC}$ for data transfer.

The capacitive coupling HBC, also called near-field coupling [31], and electrostatic coupling [17, 32] are described in Figure 2. The signal electrodes at both transmitter and receiver are attached on the human skin, while the ground electrodes are floating in air. A large electric field $E t$ is introduced to the human body via the signal electrode of transmitter [31]. The conductivity of human body not only couples electric field to environment (i.e., Ee) but also serves as a conducting plate that induces electric fields (i.e., $E a$, $E c$, and $E d$ ) to other conducting plates (ground electrodes) $[33,34]$. The return path of the transmitted signal is formed by the electric fields $E a, E c, E e-E b, E r-E f-E b$, and $E d-E f-E b$. In other words, the electric fields in environment or through external earth ground serve as the return path [35]. At the receiver, the received signal as electric potential difference between $E r, E f$, and $E d$ is allowed to be detected as $E r$ is much larger than $E f$ and $E d$ (conductivity of human body is much higher than that of air) [34]. On the other hand, the electric potential difference is small due to the escaped electric fields $E e, E a$, and $E c$. Furthermore, the detected signal is unstable and highly influenced by the environment on accounting that the electric fields $E f$ and $E e$ vary along with environment changes [17] (e.g., the appearance of metallic furniture, wires, water, and office equipment will change the backward capacitance [36]).

To avoid the human body's antenna effect of radiating signal $(\lambda / 2$ dipole is around $1 \mathrm{~m})$, the operating frequency should be lower than $150 \mathrm{MHz}$ [31]. And most of the electric field will concentrate around the tip of arm wearing transmitter [19]. Capacitive coupling HBC is suitable for those applications requiring higher operating frequencies (approximately tens of $\mathrm{MHz}$ ) and longer body transmission distances (usually along the whole body). Nevertheless, capacitive coupling $\mathrm{HBC}$ is vulnerable to external interferences and other unpredictable effects, such as the subjects' movements [37].

2.2.2. Galvanic Coupling HBC. The galvanic coupling HBC was firstly reported in 1997 by the Japanese researchers Handa et al. [38]. The ECG signal from the chest was modulated into micro-Ampere electric current, coupled into human body by electrodes, and detected by a pair of receiving electrodes on the wrist. The transmitting and receiving electrodes were in direct contact with the body, resulting in galvanic coupling signal transmission. This system worked with small power consumption, only $8 \mu \mathrm{W}$. The results suggest that data transmission with low transmission power is possible by using galvanic coupling $\mathrm{HBC}$.

In 1998, Lindsey et al. [39] tested the galvanic coupling HBC between an implantable device and an external data acquisition system. In the system, two platinum electrodes (each $0.38 \mathrm{~mm}$ in diameter, separated by $2.5 \mathrm{~mm}$ ) were used to inject sinusoidal currents with frequencies of $2-160 \mathrm{kHz}$ and amplitudes of 1-3 mA into the leg of a human cadaver. EMG electrodes on the surface of the leg were deployed to detect the voltage differences. The human body channel resulted in attenuation within $37-47 \mathrm{~dB}$. This prototype 


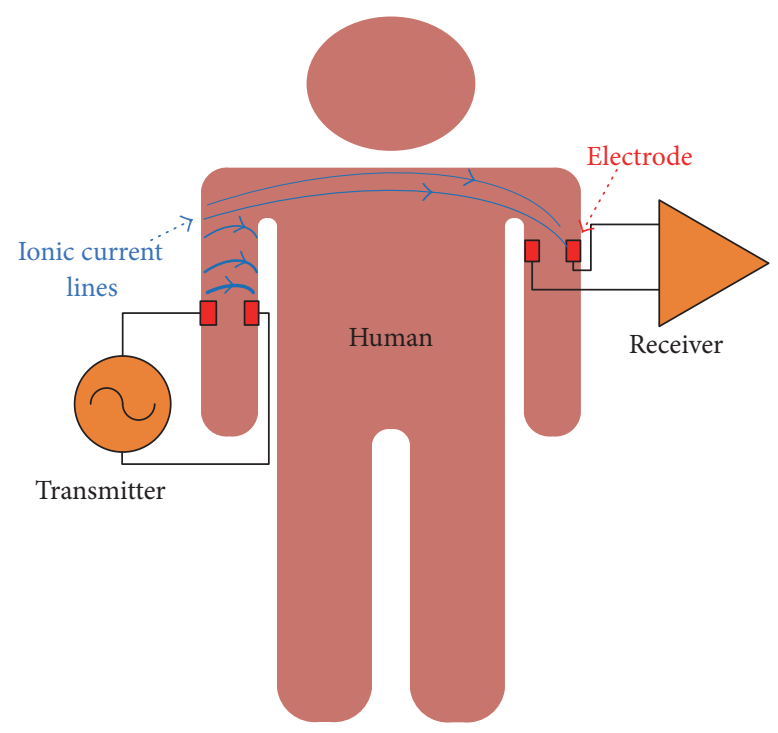

Figure 3: Principle of galvanic coupling HBC.

demonstrates that it is feasible to utilize galvanic coupling $\mathrm{HBC}$ for data transmission between implantable device and body surface mounted sensors.

Galvanic coupling $\mathrm{HBC}$, also termed waveguide $\mathrm{HBC}$ [40], utilizes the ionic fluids in body and volume conduction properties of tissues [39] to convey electrical signal. The simplified model is depicted in Figure 3. A major ionic current is generated within the tissue close to the transmitter. With a separation distance to the transmitter, the current will decrease due to the impedance of human tissue. The small current induces electric potential that can be detected at the receiver by a high-gain differential amplifier. The existing experiments or prototypes are usually performed on human limb. Since the signal propagation principle is based on the ionic current, the operation frequency should be low (i.e., $<1 \mathrm{MHz}$ [41]). And in such low frequency, the signal radiation or leakage into the space can be negligible [20].

Compared with capacitive coupling $\mathrm{HBC}$, galvanic coupling $\mathrm{HBC}$ operates in lower frequency and is less influenced by the environment, which makes the signal transmission much more stable and reliable. Therefore, galvanic coupling $\mathrm{HBC}$ is more suitable for the vital physiological signal transmission, especially for implantable device communication. Certainly, it trades off data rate. Fortunately, the data rate requirements for the vital physiological signal transmission are relatively low, for example, $75 \mathrm{kbps}$ in ECG, $1.6 \mathrm{kbps}$ in $\mathrm{SpO} 2$, and $<100 \mathrm{kbps}$ for both pacemaker and implantable glucose sensors [42]. Therefore, galvanic coupling HBC is also a promising candidate for the vital physiological data transmission among wearable and implantable devices.

\section{Electrical Properties of Human Tissues and HBC Signal Propagation Models}

After the pioneering work of successfully implementing $\mathrm{HBC}$, considerable researches have been conducted by

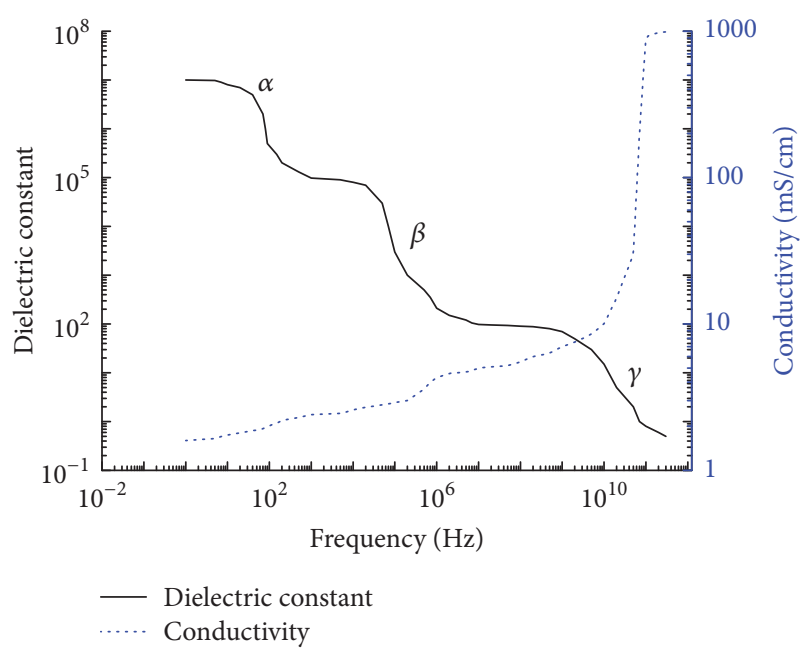

Figure 4: Dielectric constant and conductivity of tissue and cell [48].

research groups to investigate the channel gain at different distances and regions of body such as arm, thorax, leg, and the back $[43,44]$. Accompanying with the experimental investigations, the theoretical researches on the transmission mechanism have become a major research topic. The signal propagation model includes numerical model and analytical model. The numerical method can achieve more accurate calculation for the realistic structure and geometric of human body, but sacrificing computation time and cost. The numerical model for HBC includes the finite difference time domain (FDTD) simulation model (e.g., [19]) and finite element method (FEM) simulation method (e.g., [20, 35, 41]) model. On the other hand, the analytical model saves computation time but is limited to simple structures such as circle and cylinder. The analytical model mainly includes the simplified circuit model [31, 41, 44, 45], asymmetric distributed circuit model [37, 46], and quasistatic field model [47].

3.1. Electrical Property of Tissues. The signal propagation models in HBC are generally based on the electrical properties of human tissues. The frequency dispersion mechanism of human tissue was first introduced by Schwan [48]. Three main dispersion regions denoted by $\alpha, \beta$, and $\gamma$ region at respective frequencies of low frequency range, RF range, and gigahertz frequency range, response for the behavior of tissue. As depicted in Figure 4, the three dispersion regions are as follows.

$\alpha$ Dispersion (Low $\mathrm{kHz}$ ). It is not known well and is related to the polarization of counterion atmosphere near charge surfaces in the tissue or polarization of large membrane-bound structures in the tissue. Or it is associated with ionic diffusion process at the site of cellular membrane. It is apparent in permittivity and hardly noticeable in conductivity.

$\beta$ Dispersion $(0.1-10 \mathrm{MHz})$. Relaxation effects are caused by proteins, lesser extended amino acid residues, and the bioimpedance (capacitance of membrane and resistance of 


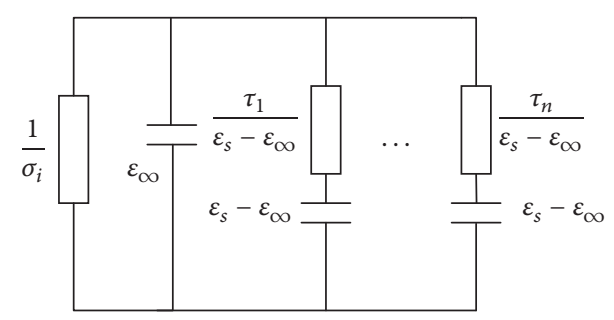

Figure 5: Circuit model of the tissue (multiple Cole-Cole model).

fluid) of organelles inside the cell, cell nuclei, and mitochondria. As the cell membranes have negligible impedance, charging of cell membranes through intracellular and extracellular becomes easier. The current passes through the extracellular and intracellular media; therefore, the conductivity becomes higher.

$\gamma$ Dispersion (Above $1 \mathrm{GHz}$ ). High conductivity region is mainly caused by a variety of tissues (amino acids, nucleic) and protein solution.

Based on many reported experimental data of tissues, the Cole-Cole equation is adopted to predict the change of dielectric properties of a tissue over frequency:

$$
\varepsilon^{*}(\omega)=\varepsilon_{\infty}+\frac{\varepsilon_{s}-\varepsilon_{\infty}}{1+\left(j \omega \tau_{n}\right)^{1-a_{n}}},
$$

where $\tau$ is the relaxation time constant of the polarization mechanism in the relaxation regions and $\varepsilon_{\infty}$ and $\varepsilon_{s}$ are the permittivity at frequencies $\omega \tau \gg 1$ and $\omega \tau \ll 1$, respectively. Equation (1) encompasses a frequency independent part $\varepsilon_{\infty}$ due to ion conduction and a frequency dependent part related to dielectric relaxation. However, the dispersion region may be broadened by many mechanisms due to the complexity of both the structure and composition of biological material. The tissue spectrum may therefore be more appropriately described by multiple Cole-Cole dispersion:

$$
\varepsilon^{*}(\omega)=\varepsilon_{\infty}+\sum_{n} \frac{\varepsilon_{s}-\varepsilon_{\infty}}{1+\left(j \omega \tau_{n}\right)^{1-a_{n}}}+\frac{\sigma_{i}}{j \omega \varepsilon_{0}}
$$

where $\sigma_{i}$ is the static ionic conductivity. The complex conductivity and impedance of tissue are calculated by

$$
\begin{aligned}
\sigma^{*} & =j \omega \varepsilon_{0} \varepsilon^{*}, \\
z^{*} & =\frac{1}{\sigma^{*}} .
\end{aligned}
$$

Based on (2) and (3), the electrical properties of tissue can be modelled by equivalent circuit with conventional resistors and capacitors as shown in Figure 5.

\subsection{HBC Signal Propagation Models}

3.2.1. Circuit Model. Wegmueller [41] developed a simple four-terminal circuit model with six body tissue impedances and four electrode-skin impedances for low frequency $(<1 \mathrm{MHz})$ galvanic coupling HBC. The simplified circuit

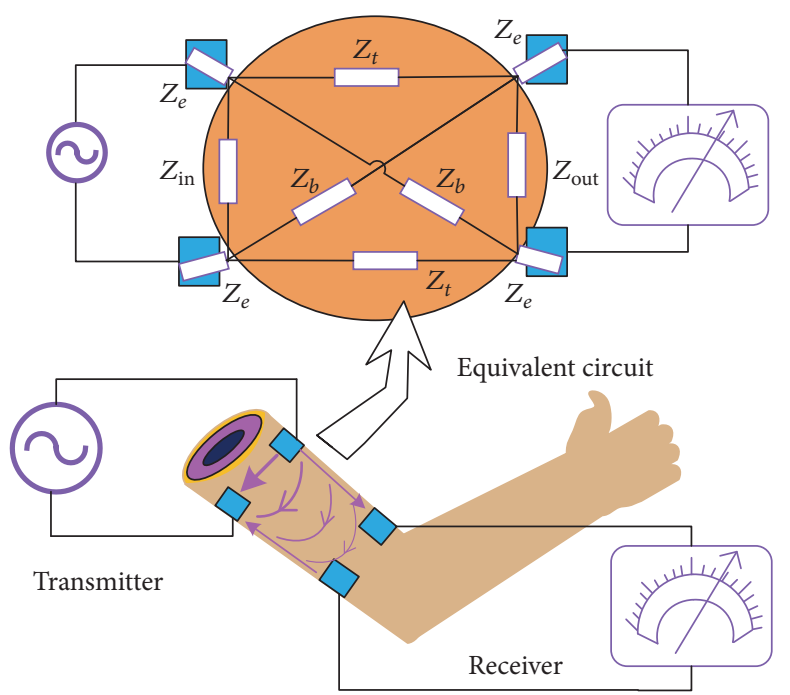

Figure 6: Simplified circuit model for galvanic coupling HBC.

model is shown in Figure 6. This model takes into account the impedance of human limb, such as longitudinal $\left(Z_{t}\right)$ and cross $\left(Z_{b}\right)$ channel impedances, as well as electrode-skin impedance $\left(Z_{e}\right)$, input $\left(Z_{\text {in }}\right)$ and output impedances $\left(Z_{\text {out }}\right)$. The channel transfer function can be described as follows:

$$
\begin{aligned}
& H=\frac{Z_{\text {out }}\left(Z_{b}-Z_{t}\right)}{2 Z_{t} Z_{b}+Z_{\text {out }} Z_{b}+Z_{\text {out }} Z_{t}} \\
& H_{\text {total }} \\
& \quad=\frac{H}{1+Z_{e}\left[1 / Z_{t}+1 / Z_{b}+H\left(1 / Z_{b}+1 / Z_{\text {in }}-2 / Z_{t}\right)\right]} .
\end{aligned}
$$

Besides the simplified circuit model, Wegmueller [41] described the body impedance by equivalent parallel circuit of resistance and capacitance according to the Cole-Cole reference model. Then a more complex layered tissue (skin, muscle, and bone) model was developed. Each layered tissue was composed by transversal and longitudinal impedances with Cole-Cole circuits connected in a mesh. It was reported that a smaller portion of current would flow through the skin layer between the electrodes, whereas the majority would be conducted by the deeper muscle tissue.

The circuit model of skin for both capacitive coupling and galvanic coupling HBC was developed by Amparo Callejón et al. $[37,46]$. In the model, the equivalent circuit model of skin can be seen as a lossy transmission line without inductive element. And for capacitive coupling method, the model parameter (propagation constant and characteristic impedance) also depends on the capacitance effect from the external ground return path [37]. It was reported that attenuation increased up to $200 \mathrm{kHz}$ and remained approximately constant for higher frequencies in galvanic coupling HBC. For capacitive HBC, a bandpass profile was observed within 1 100 MHz. The circuit model for these two coupling methods was also addressed in [44], and it was found that two-electrode arrangement achieved higher gain than the four-electrode arrangement. 
Recently, Kibret et al. [45] developed an equivalent circuit model with subject specific anthropometric measures. The gain was found to rise slowly as frequency increased from $200 \mathrm{kHz}$ to $10 \mathrm{MHz}$, and phase fell from $50^{\circ}$ to $10^{\circ}$ as frequency increased.

3.2.2. Finite Difference Time Domain (FDTD) Method. Fujii et al. utilized the FDTD method to calculate the electric field distribution in human body $[19,49,50]$. It was found that most part of the electric field was concentrated around the tip of the arm, and biological tissue-equivalent solid phantom was sufficient to mimic the human body [19]. Similar results were found in [51] that dominant signal was on the surface of the arm.

3.2.3. Finite Element Method (FEM) Method. Xu et al. [35] proposed a circuit coupled FEM model for capacitive coupling HBC. The human body was considered as a circuit of lumped resistors paralleled with lumped capacitors. The channel gain satisfied high-pass profile and the return path capacitance was dependent on the transceiver ground, as well as external ground. Recently, Callejon et al. [20] investigated the electric field distribution and current density using FEM. It is found that the electric field is mainly confined to the outer layer of the arm, and the radiation could be neglected. The majority of electric current in the galvanic coupling $\mathrm{HBC}$ travels through muscle.

3.2.4. Analytical Electromagnetic Model. The analytical electromagnetic model is developed by solving Maxwell's equations and specific boundary conditions. A theoretical model, wherein the electric field was assumed to be composed of near field, inductive field, and far-field, was proposed by Bae et al. [52] for the capacitive coupling HBC. The quasistatic field modelling of galvanic coupling $\mathrm{HBC}$ was developed by Pun et al. [47, 53]. The channel gain follows the high-pass characteristic in sub- $\mathrm{MHz}$, and the signal radiation into space is negligible.

The summary of signal propagation models and their main results are depicted in Table 2 . From the table, the signal propagation models are based on the static body behavior. While for dynamic body behavior, the signal propagation model is not yet developed.

\section{Channel Characteristics and Communication Performance of HBC Systems}

Besides the signal propagation model, one important issue that related to data transmission is the channel characteristic, which generally guides us to select the suitable transmission parameters, such as frequency band and modulation methods. In what follows, the channel characteristics are discussed.

\subsection{Channel Characteristics and Transmission Parameters.} From the main results of signal propagation model, the channel gain is generally analyzed. The channel gain behaves generally as bandpass [37] or high-pass [31, 45]. Besides, the channel characteristics in static body behavior that in dynamic body behavior are of important. That is due to the fact that HBC system permits the doctors or physicians to access the physiological data without interrupting the patients' everyday life, in which the human body moves and posts different gestures (such as grasping cup to drink), which is expected to affect the data transmission. From the experimental investigation, it was found that the large scale body behavior, such walking, sitting, and standing, has little effect on the channel $[54,55]$. The joint behavior, such as joint flexion or extension, can cause gain variate in $2 \sim 5 \mathrm{~dB}[56,57]$, while phase is little affected [57]. The channel characteristics' analysis is shown in Table 3. As shown in Table 3, the other channel characteristics such as phase, linearity of channel, and noise in channel are little discussed. From the recent investigation, the phase in galvanic coupling $\mathrm{HBC}$ channel follows a decreased trend $[45,58]$, while the noise in the channel is assumed to be mainly from electronic components [41] and electrode-skin interface [59], which are assumed to follow the additive white Gaussian noise (AWGN) property.

The transmission parameters, which are either for testing conditions of experiments or specifications (i.e. narrow band or wideband) that they chose for the demo system or for achieving the best performance, are generally selected based on the results of channel gain profile. The parameters' selections in some typical literatures are summarized in Table 4. The transmission parameters such as carrier frequency and modulation method, which are involved in the physical layer design, have been selected based on the empirical results and analysis of channel characteristics. For instance in [17], the carrier frequency and bandwidth were determined via the $-3 \mathrm{~dB}$ frequency response. In [32], $10 \mathrm{MHz}$ was suggested to be the suitable carrier frequency due to its maximum channel gain. Similarly in [60], suitable frequency range (from $200 \mathrm{MHz}$ to $600 \mathrm{MHz}$ ) for transmission was determined corresponding to the lowest path loss. Owing to the relative flat and high channel gain, a carrier frequency of $256 \mathrm{kHz}$ and bandwidth of $100 \mathrm{kHz}$ were chosen in [41]; meanwhile the transmission power was averagely allocated within this band. To show the feasibility of achieving biomedical applications in clinical trials, BPSK and QPSK were selected to be implemented in the HBC prototype [61]. QPSK was chosen as suitable modulation method based on the results of hardware complexity analysis [41]. Based on the comparison measurements, BPSK was selected as the optimal modulation method among BPSK, QPSK, MSK, and 16QAM [62]. From Table 4, the transmission parameters' selection is generally based on the empirical and scattering measurement results. Recent investigation [63] offers an innovative view to select the transmission parameter based on the theory of channel capacity; however, further experimental verification of the theory and analysis is needed.

4.2. Communication Performance of HBC Systems. The communication performance of $\mathrm{HBC}$ systems was investigated by using simulations [37] and experiments [17, 61, 64, 65]. Also many prototypes $[32,41,60]$ have been developed to achieve different communication performance. Various transmission parameters (carrier frequency, modulation scheme, and 
TABLE 2: Signal propagation models and results.

\begin{tabular}{|c|c|c|c|}
\hline Model & Coupling method & Model mechanism & Main results \\
\hline $\begin{array}{l}\text { Equivalent circuit model, Hachisuka et al. } \\
\text { [44] }\end{array}$ & $\begin{array}{l}\text { Cap-HBC \& } \\
\text { Gal-HBC }\end{array}$ & $\begin{array}{l}\text { Four-terminal circuit } \\
\text { model with six } \\
\text { impedances }\end{array}$ & Higher gain in Cap-HBC \\
\hline Multilayer tissue model, Wegmueller [41] & Gal-HBC & $\begin{array}{l}\text { Equivalent Cole-Cole } \\
\text { circuits }\end{array}$ & Muscle conduct majority current \\
\hline $\begin{array}{l}\text { Distributed circuit model, Amparo Callejón } \\
\text { et al. [37] }\end{array}$ & $\begin{array}{l}\text { Cap-HBC \& } \\
\text { Gal-HBC }\end{array}$ & Lossy transmission line & $\begin{array}{l}\text { Cap-HBC: bandpass gain in } 1-100 \mathrm{MHz} \\
\text { Gal-HBC: peak gain in } 20-50 \mathrm{kHz}\end{array}$ \\
\hline Circuit model, Kibret et al. [45] & Gal-HBC & $\begin{array}{l}\text { Simplified layered tissue } \\
\text { circuits }\end{array}$ & High-pass profile in gain in $0.2-10 \mathrm{MHz}$ \\
\hline Distributed RC model, Cho et al. [31] & Cap-HBC & $\begin{array}{l}\text { Cascaded blocks of RC } \\
\text { circuit }\end{array}$ & High-pass profile in gain in $0.1-100 \mathrm{MHz}$ \\
\hline FEM model, Xu et al. [35] & Cap-HBC & $\begin{array}{l}\text { Body path with } \pi \text { circuit } \\
\text { model }\end{array}$ & $\begin{array}{l}\text { High-pass gain in } 10-100 \mathrm{MHz} \text {, body can } \\
\text { reduce return path capacitance }\end{array}$ \\
\hline FEM model, Callejon et al. [20] & Gal-HBC & $\begin{array}{l}\text { Time-harmonic } \\
\text { charge-continuity } \\
\text { equation, Gauss' law }\end{array}$ & $\begin{array}{c}\text { Electric field mainly in outer layer of arm, } \\
\text { electric current mainly in muscle layer, } 20 \mathrm{~dB} \\
\text { attenuation with additional } 5 \mathrm{~cm} \text { channel } \\
\text { length }\end{array}$ \\
\hline FDTD model, Fujii et al. [19] & Cap-HBC & FDTD & $\begin{array}{l}\text { TX GND electrode strengthen signal, electric } \\
\text { field confined at the tip of arm }\end{array}$ \\
\hline Quasistatic field model, Pun et al. [47] & Gal-HBC & $\begin{array}{l}\text { Quasistatic field, } \\
\text { Maxwell's equation }\end{array}$ & High-pass gain in sub-MHz \\
\hline Electromagnetic field model, Bae et al. [52] & Cap-HBC & $\begin{array}{l}\text { Time harmonic } \\
\text { electromagnetic field } \\
\text { Maxwell's equation }\end{array}$ & $\begin{array}{c}\text { Near-field region: signal attenuation depend } \\
\text { on }(1 / r)^{3} \text {, far field region: signal attenuation } \\
\text { satisfy } 1 / r\end{array}$ \\
\hline
\end{tabular}

TABLE 3: Channel characteristics from the literature.

\begin{tabular}{|c|c|c|c|c|}
\hline Gain & Phase & Linearity of channel & Noise in channel & Gain \\
\hline \multicolumn{5}{|c|}{ Capacitive coupling HBC } \\
\hline \multirow{4}{*}{ Static body behavior } & Bandpass profile [31] & \multirow{4}{*}{ Non } & \multirow{4}{*}{ Non } & \multirow{4}{*}{ Non } \\
\hline & Influence by electrode [35] & & & \\
\hline & Better results in limbs [54] & & & \\
\hline & TX GND electrode strengthen signal [19] & & & \\
\hline \multirow{2}{*}{ Dynamic body behavior } & Movement sensitive $[54,55]$ & \multirow{2}{*}{ Non } & \multirow{2}{*}{ Non } & \multirow{2}{*}{ Non } \\
\hline & Joint flexion cause gain variate in $2 \mathrm{~dB}$ [96] & & & \\
\hline \multicolumn{5}{|c|}{ Galvanic coupling HBC } \\
\hline Static body behavior & $\begin{array}{l}\text { Higher gain in trunk and back [54] } \\
\text { High-pass profile [45] } \\
\text { High-pass profile [59] }\end{array}$ & Decrease [45] & Linear channel [59] & $\begin{array}{c}\text { AWGN } \\
{[41,59,92]}\end{array}$ \\
\hline Dynamic body behavior & $\begin{array}{c}\text { Not sensitive in sitting \& waling [54] } \\
\text { Increase as joint flexion [56] } \\
\text { Joints and biceps muscle have a great effect } \\
{[57]}\end{array}$ & $\begin{array}{l}\text { Decrease trend [57] } \\
\text { Not sensitive by } \\
\text { movement [57] }\end{array}$ & Non & AWGN [57] \\
\hline
\end{tabular}

transmission power) were adopted to achieve different data rates.

For capacitive coupling $\mathrm{HBC}$, narrowband modulation on-off keying $(\mathrm{OOK})$ and wideband signaling direct sequence spread spectrum (DSSS) were examined in [17]. The OOK was found to be more effective and easier to be implemented, and a data rate of $2.4 \mathrm{kbps}$ was obtained with carrier frequency $330 \mathrm{kHz}$ and bandwidth $400 \mathrm{kHz}$. The data rate was extended to $9.6 \mathrm{kbps}$ by using frequency shift keying (FSK) modulation in $[66,67]$. A higher data rate of $38.4 \mathrm{kbps}$ was achieved with carrier frequency of $160 \mathrm{kHz}$ in [68]. Since 2006, experiments sprung up to obtain data rate around Mbps. According to Ruiz and Shimamoto [69], the received power could be modelled as a normal distribution. The modulation methods BPSK, QPSK, 8PSK, 16QAM, and MSK provided symbol rates range from $100 \mathrm{ksps}$ to $5 \mathrm{Msps}$ with transmission power of $15 \mathrm{dBm}$ and carrier frequency of $200 \mathrm{MHz}[21,65]$. Although MSK achieved the best performance [65], it was the most sensible modulation scheme so that the modulation quality decreased as symbol rate 
TABLE 4: Transmission parameters selection from literatures.

\begin{tabular}{|c|c|c|c|c|c|}
\hline \multirow{2}{*}{ Literature } & \multicolumn{2}{|c|}{ Suitable carrier frequency } & \multicolumn{2}{|c|}{ Suitable modulation method } & \multirow{2}{*}{ Objective of selection } \\
\hline & Frequency & Guideline & Modulation & Rule & \\
\hline Zimmerman [17] & $300 \mathrm{kHz}$ & $-3 \mathrm{~dB}$ band & OOK & Simple & Test narrow band transmission \\
\hline Hachisuka et al. [32] & $10.7 \mathrm{MHz}$ & $\begin{array}{l}\text { Maximum } \\
\text { gain }\end{array}$ & FSK & Simple & Minimize power consumption \\
\hline $\begin{array}{l}\text { Ruiz and Shimamoto } \\
{[65]}\end{array}$ & $200 \mathrm{MHz}$ & $\begin{array}{l}\text { Maximum } \\
\text { gain }\end{array}$ & MSK & Empirical results & $\begin{array}{l}\text { Find suitable modulation } \\
\text { method }\end{array}$ \\
\hline Wegmueller [41] & $256 \mathrm{kHz}$ & High gain & QPSK & Hardware complexity & Test HBC transceiver \\
\hline Wegmueller et al. [61] & $128 \mathrm{kHz}$ & High gain & BPSK, QPSK & Empirical results & Test clinical trials in transceiver \\
\hline Zhang et al. [62] & $10.7 \mathrm{MHz}$ & $\begin{array}{l}\text { Maximum } \\
\text { gain }\end{array}$ & BPSK & Empirical results & Determine optimal modulation \\
\hline Lin et al. [60] & $200-600 \mathrm{MHz}$ & Low path loss & OOK & Saving power & Optimization of SoC chip \\
\hline
\end{tabular}

increased [21]. A data rate of $5 \mathrm{Mbps}$ was achieved in [70] by using OOK/FSK modulation. By using the delay-lockedloop based BPSK demodulator, a data rate between $164 \mathrm{kbps}$ and $1.313 \mathrm{Mbps}$ was achieved with transmission power of $1.4 \mathrm{~mW}$ in the HBC transceiver developed by Cho et al. [71]. Later, a $65 \mathrm{~nm}$ CMOS process HBC transceiver [72] for both entertainment and healthcare was implemented by this research group; for the latter case, a data rate of $100 \mathrm{kbps}$ was achieved with low transmission power of $21 \mu \mathrm{W}$. By using a wideband pulse system on chip (SOC) transceiver was implemented by Song et al. [73], and the chip obtained a data rate of $2 \mathrm{Mbps}$ within the frequency band from $10 \mathrm{kHz}$ to $100 \mathrm{MHz}$. By using DSSS and frequency hopping FSK modulation, the data rate was extended to $10 \mathrm{Mbps}$ in [74] and in [75]. With other wideband modulation such as frequency selective Walsh spread and UWB, the data rates were up to $2 \mathrm{Mbps}[76]$ and $5 \mathrm{Mbps}[77,78]$. Recently, a wideband signaling $\mathrm{HBC}$ transceiver with high data rate between 1 Mbps to 40 Mbps was developed by Chung et al. [79].

For galvanic coupling HBC, the first prototype achieved only $0.9 \mathrm{kbps}$ by employing pulse width modulation (PWM) [38]. A data rate of $9.6 \mathrm{kbps}$ was obtained by using FSK modulation with carrier frequency of $10.7 \mathrm{MHz}[32,40]$. The new component electrooptic sensor was introduced in the transceiver by Shinagawa et al. [80]; a data rate of $10 \mathrm{Mbps}$ was reached. The comparison of modulation schemes FSK and BPSK was conducted by Wegmueller et al. [43]. It was found that, with input current of $2 \mathrm{~mA}$, BPSK and FSK obtained a data rate of $255 \mathrm{kbps}$ and $128 \mathrm{kbps}$, respectively. With a SNR of $6 \mathrm{~dB}$ [61], both BPSK and QPSK achieved a data rate of $64 \mathrm{kbps}$ and BER of $10^{-4}$. BPSK and QPSK were the suitable modulation schemes among PSK, FSK, and QAM [41] in galvanic coupling HBC. According to Zhu et al. [64], a minimum transmission power of $-17.8 \mathrm{dBm}$ was utilized to achieve data rate of $10 \mathrm{Mbps}$ and BER of $10^{-6}$. The SOC prototype from Lin et al. [60] obtained a data rate of $2 \mathrm{Mbps}$ by using $\mathrm{OOK}$ with transmission power $-4 \mathrm{dBm}$.

The data rates in the experiments and prototypes are presented and compared in Table 5. There is a general trend that the data rate increases from kbps to Mbps since 2000. Generally, the transmission distance of capacitive coupling HBC system is in several tens to even hundreds of centimeters. However, galvanic coupling HBC transmits with shorter distance around tens of centimeter and lower data rate than capacitive coupling HBC. It is noted that capacitive coupling $\mathrm{HBC}$ can transmit with wideband signaling, which can obtain high data rate but requires large bandwidth.

4.3. Applications of $H B C$. The HBC prototypes have been applied in the healthcare data transmission. For instance, Handa et al. [38] developed a galvanic coupling HBC prototype to transmit the ECG signal from chest to limb. Later, a prototype [40] of galvanic coupling $\mathrm{HBC}$ has been applied to transmit the heart rate and SpO2. Yoo et al. [81] implemented a HBC system to transmit the ECG and blood pressure. And a SOC chip for transferring the ECG signal was developed by Lin et al. [60].

Recently, the channel characteristics in HBC, such as gain and phase, were found to have great relevance with some physiological parameter, such as muscle contraction or relaxation, body fluid hydration, and dehydration. Based on these principles, the galvanic coupling $\mathrm{HBC}$ system has been adopted to conduct some physiological parameter measurement. For instance, the qualitative hydration and dehydration rate affected by the physiological state and metabolic equilibrium can be accessed by the galvanic coupled HBC signal propagation [82]. This mechanism has been utilized to evaluate the body fluid [82] and help to aid diagnosis and treatment of fluid disorders such as lymphoedema. According to Chen et al. [57], the gain variation has great relevance with the joint flexion. The relationship between joint angle and channel gain variation has been accessed by using the polynomial curve fitting method [83]. The obtained relationship is then adopted to perform the joint angle estimation [83], which can be applied in prosthesis control and gait analysis.

\section{Safety Considerations and Experimental Issues}

During in vivo $\mathrm{HBC}$ experiment, the electric signals are applied into the human body by the electrodes, which would pose potential health risks to the human body, such as the danger of electrical shock and safety issue of using electrodes. 
TABLE 5: Communication performance of HBC system.

\begin{tabular}{|c|c|c|c|c|c|}
\hline Literature (authors, year) & TX power & Channel capacity & Data rate & TX distance & Modulation method \\
\hline \multicolumn{6}{|c|}{ Capacitive coupling HBC } \\
\hline Zimmerman [17], 1995 & - & $417 \mathrm{kbps}$ & $2.4 \mathrm{kbps}$ & - & OOK \\
\hline Post et al. [66], 1997 & $20 \mathrm{dBm}$ & $50 \mathrm{kbps}$ & $9.6 \mathrm{kbps}$ & $200 \mathrm{~cm}$ & FSK \\
\hline Matsushita et al. [67], 2000 & - & - & $9.6 \mathrm{kbps}$ & - & FSK \\
\hline Partridge et al. [68], 2001 & - & - & $38.4 \mathrm{kbps}$ & $200 \mathrm{~cm}$ & FSK \\
\hline Shinagawa et al. [80], 2004 & - & - & $10 \mathrm{Mbps}$ & $150 \mathrm{~cm}$ & - \\
\hline Song et al. [73], 2006 & - & - & $2 \mathrm{Mbps}$ & - & Wideband \\
\hline Zhu et al. [64], 2009 & $-14 \mathrm{dBm}$ & - & $10 \mathrm{Mbps}$ & $15 \mathrm{~cm}$ & QPSK \\
\hline Cho et al. [75], 2009 & $-5 \mathrm{dBm}$ & - & $10 \mathrm{Mbps}$ & $120 \mathrm{~cm}$ & Wideband \\
\hline Zhang et al. [62], 2010 & - & - & $0.1 \sim 10 \mathrm{Mbps}$ & $10 \sim 150 \mathrm{~cm}$ & BPSK \\
\hline Nie et al. [70], 2012 & $-15 \sim 5 \mathrm{dBm}$ & - & $5 \mathrm{Mbps}$ & $10 \sim 50 \mathrm{~cm}$ & $\mathrm{OOK}$ \\
\hline Hyoung et al. [78], 2012 & - & - & $2 \mathrm{Mbps}$ & $170 \mathrm{~cm}$ & Wideband \\
\hline Cho et al. [71], 2014 & $1.4 \mathrm{dBm}$ (or $1.4 \mathrm{~mW}$ ) & - & $164 \mathrm{kbps} \sim 1.313 \mathrm{Mbps}$ & - & BPSK \\
\hline Cho et al. [72], 2015 & $-16.7 \mathrm{dBm}($ or $21 \mu \mathrm{W})$ & - & $100 \mathrm{kbps}$ & - & OOK \\
\hline Chung et al. [79], 2016 & - & - & $1 \mathrm{Mbps} \sim 40 \mathrm{Mbps}$ & - & Wideband \\
\hline \multicolumn{6}{|c|}{ Galvanic coupling HBC } \\
\hline Handa et al. [38], 1997 & $-21 \mathrm{dBm}$ & - & $0.9 \mathrm{kbps}$ & $40 \mathrm{~cm}$ & PWM \\
\hline Hachisuka et al. [40], 2003 & $-10 \mathrm{dBm}$ & - & $9.6 \mathrm{kbps}$ & $28 \mathrm{~cm}$ & FSK \\
\hline Wegmueller et al. [43], 2005 & $-3 \mathrm{dBm}$ & - & $255 \mathrm{kbps}$ & $10 \sim 15 \mathrm{~cm}$ & BPSK \\
\hline Ruiz and Shimamoto [65], 2006 & $15 \mathrm{dBm}$ & - & $2 \mathrm{Mbps}$ & $20 \mathrm{~cm}$ & QPSK \\
\hline Wegmueller [41], 2007 & $-6 \mathrm{dBm}$ & $1.23 \mathrm{Mbps}$ & $64 \mathrm{kbps}$ & $10 \sim 25 \mathrm{~cm}$ & QPSK \\
\hline Lin et al. [60], 2011 & $-30 \sim-4 \mathrm{dBm}$ & - & $2 \mathrm{Mbps}$ & - & OOK \\
\hline Li et al. [92], 2013 & $-5 \mathrm{dBm}$ & - & $200 \mathrm{kbps}$ & $6 \mathrm{~cm}$ & QPSK \\
\hline
\end{tabular}

5.1. Safety Guidelines. Whenever any form of energy is introduced into the human body, it is important to understand what risks might result from applying energy to internal tissue. The most obvious effect is heating. Since a significant portion of energy is absorbed and converted into heat during signal propagation, this would potentially lead to temperature increase. More importantly, the induced current intensity at specific frequency determines the intensity of the electrical shock through the body. The applied electric current or voltage on human body must follow IEEE Std. C95.12005 [84] and International Commission on Nonionizing Radiation Protection (ICNIRP) guidelines [85]. The IEEE Std. C95.1 2005 is more detailed about the safety levels with respect to the human body exposure to radio frequency electromagnetic field. For instance, the restriction of electric field between $100 \mathrm{kHz}$ and $3 \mathrm{GHz}$ within localized human body such as extremities and pinnae should be $4^{c} \mathrm{~W} / \mathrm{kg}$ (where $c$ is defined as tissue volume in the shape of a cube) [84]. The maximum harmless induced current in HBC should be lower than $20 \mathrm{~mA}$ at frequency range of $100 \mathrm{kHz}$ to $110 \mathrm{MHz}$ (general public exposure) [85].

5.2. Electrodes. HBC operates without the antenna; instead the electrodes are used to couple the signal into the human body. From the literature, the $\mathrm{Ag} / \mathrm{AgCl}$ electrodes are generally utilized in the $\mathrm{HBC}$ systems $[44,54,60,86]$. For $\mathrm{Ag} / \mathrm{AgCl}$ electrode, the major process that occurs at the electrodeskin interface is a transformation of charge carriers between electrons and ions. And the transformation is governed by the reduction and oxidation chemical reaction [87], which can be described as follows:

$$
\begin{aligned}
\mathrm{Ag} & \longleftrightarrow \mathrm{Ag}^{+}+\mathrm{e}^{-} \\
\mathrm{Ag}^{+}+\mathrm{Cl}^{-} & \longleftrightarrow \mathrm{AgCl}
\end{aligned}
$$

The electrodes with materials safety for human body are also recommended, such as carbon/graphite electrodes [57, 59]. The Pt electrodes were used in [39], since Pt electrode is commonly used for signal stimulation [88]. Some other electrodes, such as copper and aluminium, were utilized [54].

5.3. Grounding Strategies. The results of $\mathrm{HBC}$ theoretical models or analysis are required to be verified by in vivo or in vitro experiments, in which the measurement system generally includes the earth-grounded electrical devices such as signal generator, oscilloscope, network analyzer, and spectrum analyzer. More than one ground connection path between two pieces of equipment (such as signal generator at transmitter and oscilloscope at the receiver) leads to different places of "Ground" points that are expected to be at the same potential, but actually, they may have different potentials due to the nonzero impedance of the interconnected cables. The difference of system ground impedance makes the ground potential at the receiver input BNC connector not the same as the ground of the system or transmitter. This potential difference or unwanted ground path signal can range from microvolts to as high as hundreds of millivolts. This unwanted ground path signal generally exists in parallel with the true 


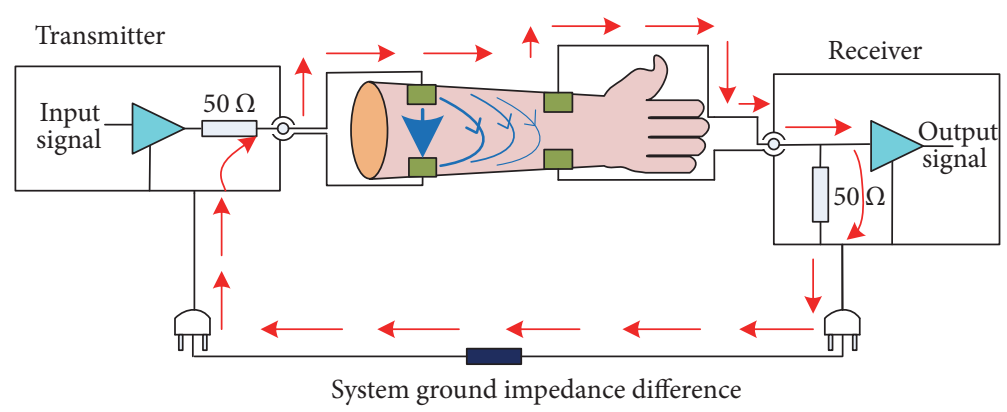

FigURE 7: Grounding problems in HBC measurement system.

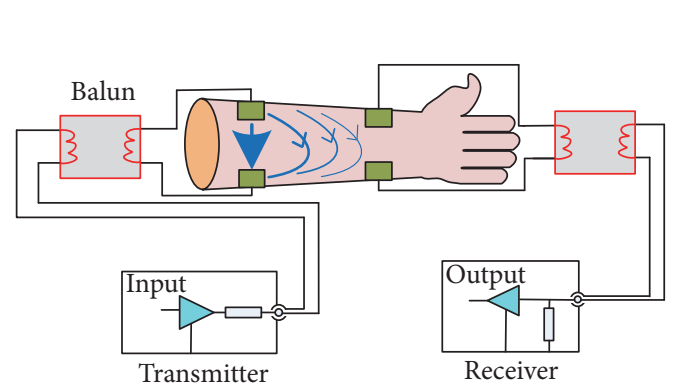

(a) With balun

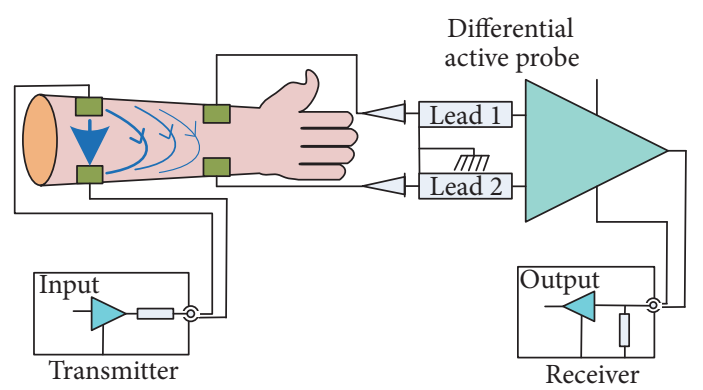

(b) With differential probe

FIGURE 8: Grounding strategy with balun transformer (a) or differential probe (b).

return ground path of HBC signal (note that this unwanted ground path signal does not exist in the real HBC system with independent battery-powered transceiver). Figure 7 illustrates the ground path signals of a typical galvanic coupling HBC measurement system. The receiver refers the measurement from the shell of the input BNC connector; the displayed waveform may not represent the real signal at the probe input, which may therefore cause incorrect measurement results at the receiver.

To minimize or reduce the unwanted ground path signal, suitable grounding strategies are required. Generally, the balun transformer or differential amplifier should be adopted. A typical measurement system with baluns at both transmitter and receiver for the galvanic coupling $\mathrm{HBC}$ is shown in Figure $8(\mathrm{a})$. In the measurement system with balun transformer, the reduction of unwanted ground path signal is influenced by the interwinding capacitance (IWC) between primary and secondary windings $[34,89,90]$. Lower IWC achieves lower measured results [89]. According to the recent investigations, the measured result is also influenced by the symmetry to ground of the balun transformer [91]. The typical measurement systems using balun are [54,64]. A setup of the measurement system with differential probe is shown in Figure 8(b). The detected potential at the respective lead of differential probe refers to the same/common ground point, which eliminates the common-mode voltage picked up by the system ground impedance. By using the differential probe, which always contains a differential amplifier with several $\mathrm{dB}$ gain, satisfactory receiving performance will be obtained. Generally, differential probe is always utilized in galvanic coupling HBC system, such as $[57,59,92]$.

\section{Conclusions and Future Studies}

The history and development of HBC have been reviewed in this paper. HBC is a short range non-RF wireless communication technique specified by the IEEE 802.15.6 using the human body as a transmission medium. In this work, we surveyed the current $\mathrm{HBC}$ coupling methods, various signal propagation models, and communication performance. Finally, the issues on in vivo experiment such as types of electrodes and grounding problems have been elaborated so that it can make the in vivo experiment safe and accurate.

From recent HBC systems, capacitive coupling HBC operates in higher frequency band, and, thus, higher data rate can be achieved. Galvanic coupling HBC operates in relatively lower frequency band and achieves lower data rate, but it has the advantages of minimizing the interference from the environment. The channel gain of HBC behaves as bandpass or high-pass in different frequency band, which indicates that a rule or guideline to select the suitable frequency band for data transmission requires to be proposed. As it stands, the HBC technique potentially offers a more power efficient and naturally secured communication method for BAN. The signal is mainly conveyed within the muscle tissue. Although HBC has many advantages, many challenges and future studies are still required to be addressed.

6.1. Channel Modelling. A suitable channel model considering the effects of joint (i.e., the structure and electrical properties of the joint) should be developed. Moreover, the change of joint angle leads to variation of channel gain; hence, developing a channel model that can cover the channel characteristics 
in dynamic body behavior would be a good research topic. Also, suitable channel model should be developed for HBC channel with longer transmission distance (i.e., the whole upper limb, from left hand to right hand). Besides the on-body channels, channel model for the implantable data transmission should be a future research topic. Moreover, the HBC channel characteristics have been evaluated on the young subjects in the literature. However, the investigation on elder people has not yet been addressed. And designing the HBC system to satisfy all the individual channel property, such as the fat, thin, young, and elder subject's HBC channel is challenging. In addition, the signal propagation models are investigated in the single channel applications, such as ECG only or blood pressure only. However, how to apply the channel model in the multiple channel applications has not been discussed yet.

6.2. System Design. Considering the HBC system design, achieving the channel capacity is challenging. Even though the multicarrier system with optimal power allocation can achieve the capacity, the multicarrier system can lead to high peak-to-average power ratio. Therefore, it is preferable to develop the single carrier system for HBC applications. Then how to design a single carrier system to achieve channel capacity and satisfy the special requirements on applications (high QoS, low power consumption, and low hardware complexity) is a future research topic.

6.3. MAC Layer Design. To build a BAN, the point-to-point data transmission is the basis. The reality BAN is generally a point-to-multipoint topology. How to apply the results on HBC channel (point-to-point data transmission) to enable the point-to-multipoint performance analysis is a future research topic. Besides the physical layer design, the design of protocol in higher layers, that is, MAC layer, should be addressed. For the network issue, the security and power management are suggested to be studied.

\section{Conflicts of Interest}

The authors declare that they have no conflicts of interest.

\section{Authors' Contributions}

Jian Feng Zhao conceived the study and helped to draft the manuscript and handled the acquisition of funding. Xi Mei Chen designed and carried out the experiments, analyzed the data, and drafted the manuscript. Bo Dong Liang helped to collect the data and draft the manuscript. Qiu Xia Chen helped to collect the data, handled the acquisition of funding, and justified the authorship. All authors have read and approved this manuscript.

\section{Acknowledgments}

The authors would like to acknowledge the financial support from Shenzhen Science and Technology Innovation Committee (nos. JCYJ20160429145314252, JCYJ20160527162817715, and JCYJ20160407160609492),
Guangdong Provincial Science and Technology Plan Projects (no. 2016A010101039), and Shenzhen Polytechnic (nos. $601522 \mathrm{k} 30007$ and 601522K30015).

\section{References}

[1] W. H. Organization, "Noncommunicable Diseases," 2015, http:// www.who.int/mediacentre/factsheets/fs355/en/.

[2] "Facts about Ageing," 2015, http://www.who.int/ageing/en/.

[3] G. Yang, L. Xie, M. Mäntysalo et al., "A health-IoT platform based on the integration of intelligent packaging, unobtrusive bio-sensor, and intelligent medicine box," IEEE Transactions on Industrial Informatics, vol. 10, no. 4, pp. 2180-2191, 2014.

[4] H.-Y. Tung, K.-F. Tsang, H.-C. Tung, K.-T. Chui, and H.-R. Chi, "The design of dual radio ZigBee homecare gateway for remote patient monitoring," IEEE Transactions on Consumer Electronics, vol. 59, no. 4, pp. 756-764, 2013.

[5] H. C. Tung, K. F. Tsang, K. L. Lam et al., "A mobility enabled inpatient monitoring system using a ZigBee medical sensor network," Sensors, vol. 14, no. 2, pp. 2397-2416, 2014.

[6] M. Patel and J. Wang, "Applications, challenges, and prospective in emerging body area networking technologies," IEEE Wireless Communications Magazine, vol. 17, no. 1, pp. 80-88, 2010.

[7] S. Li, L. D. Xu, and X. Wang, "A continuous biomedical signal acquisition system based on compressed sensing in body sensor networks," IEEE Transactions on Industrial Informatics, vol. 9, no. 3, pp. 1764-1771, 2013.

[8] M. Seyedi, B. Kibret, D. T. H. Lai, and M. Faulkner, "A survey on intrabody communications for body area network applications," IEEE Transactions on Biomedical Engineering, vol. 60, no. 8, pp. 2067-2079, 2013.

[9] K. Dam, S. Pitchers, and M. Barnard, "From pan to ban: Why body area networks?" in The Wireless World Research Forum (WWRF) Second Meeting, Nokia Research Centre, Helsinki, Finland, 2001.

[10] G. Bieber, M. Haescher, and M. Vahl, "Sensor requirements for activity recognition on smart watches," in Proceedings of the the 6th International Conference on Pervasive Technologies Related to Assistive Environments, New York, USA, New York, NY, USA, May 2013.

[11] Z. Zhang, "Photoplethysmography-based heart rate monitoring in physical activities via joint sparse spectrum reconstruction," IEEE Transactions on Biomedical Engineering, vol. 62, no. 8, pp. 1902-1910, 2015.

[12] Y. Chen, Securing wireless localization against signal strength attacks [Ph.D. thesis], The State University of New Jersey, 2007.

[13] A. Fort, J. Ryckaert, C. Desset, P. De Doncker, P. Wambacq, and L. Van Biesen, "Ultra-wideband channel model for communication around the human body," IEEE Journal on Selected Areas in Communications, vol. 24, no. 4 I, pp. 927-933, 2006.

[14] M. W. Abdullah, X. Fafoutis, E. Mellios, M. Klemm, and G. S. Hilton, "Investigation into off-body links for wrist mounted antennas in bluetooth systems," in Proceedings of the Loughborough Antennas and Propagation Conference, LAPC 2015, gbr, November 2015.

[15] M. Ghovanloo and S. Atluri, "A wide-band power-efficient inductive wireless link for implantable microelectronic devices using multiple carriers," IEEE Transactions on Circuits and Systems I: Regular Papers, vol. 54, no. 10, pp. 2211-2221, 2007.

[16] S. Mutashar, M. A. Hannan, S. A. Samad, and A. Hussain, "Analysis and optimization of spiral circular inductive coupling 
link for bio-implanted applications on air and within human tissue," Sensors, vol. 14, no. 7, pp. 11522-11541, 2014.

[17] T. Zimmerman, Personal area networks (PAN): Near-field intrabody communication, [Master, thesis], Massachusetts Institute of Technology, 1995.

[18] IEEE, "IEEE standard for local and metropolitan area networks part 15.6: Wireless body area networks, Std," 2012, https:// standards.ieee.org/findstds/standard/802.15.6-2012.html.

[19] K. Fujii, M. Takahashi, and K. Ito, "Electric field distributions of wearable devices using the human body as a transmission channel," IEEE Transactions on Antennas and Propagation, vol. 55, no. 7, pp. 2080-2087, 2007.

[20] M. A. Callejon, J. Reina-Tosina, D. Naranjo-Hernandez, and L. M. Roa, "Galvanic coupling transmission in intrabody communication: A finite element approach," IEEE Transactions on Biomedical Engineering, vol. 61, no. 3, pp. 775-783, 2014.

[21] J. A. Ruiz, J. Xu, and S. Shimamoto, "Propagation characteristics of intra-body communications for body area networks," in Proceedings of the 3rd IEEE Consumer Communications and Networking Conference (CCNC '06), vol. 1, pp. 509-513, January 2006.

[22] X. P. Yu, M. A. Do, W. M. Lim, K. S. Yeo, and J.-G. Ma, "Design and optimization of the extended true single-phase clock-based prescaler," IEEE Transactions on Microwave Theory and Techniques, vol. 54, no. 11, pp. 3828-3834, 2006.

[23] V. Manthena, Ultra low power cmos phase-locked loop frequency synthesizers [Ph.D. thesis], Nanyang Technological University, 2011.

[24] P. Chen, B. Zhao, R. Luo, and H. Yang, "A low-power alldigital GFSK demodulator with robust clock data recovery," in Proceedings of the the great lakes symposium, pp. 123-128, Salt Lake City, Utah, USA, May 2012.

[25] D. Halperin, T. Kohno, T. S. Heydt-Benjamin, K. Fu, and W. H. Maisel, "Security and privacy for implantable medical devices," IEEE Pervasive Computing, vol. 7, no. 1, pp. 30-39, 2008.

[26] L. A. Geddes, "Electronarcosis," Medical Electronics \& Biological Engineering, vol. 3, no. 1, pp. 11-26, 1965.

[27] L. A. Geddes and L. E. Baker, "The specific resistance of biological material-a compendium of data for the biomedical engineer and physiologist," Medical and Biological Engineering, vol. 5, no. 3, pp. 271-293, 1967.

[28] C. Gabriel, S. Gabriel, and E. Corthout, "The dielectric properties of biological tissues: I. Literature survey," Physics in Medicine and Biology, vol. 41, no. 11, pp. 2231-2249, 1996.

[29] S. Gabriel, R. W. Lau, and C. Gabriel, "The dielectric properties of biological tissues: II. Measurements in the frequency range $10 \mathrm{~Hz}$ to $20 \mathrm{GHz}$," Physics in Medicine and Biology, vol. 41, no. 11, pp. 2251-2269, 1996.

[30] S. Gabriel, R. W. Lau, and C. Gabriel, "The dielectric properties of biological tissues: III. Parametric models for the dielectric spectrum of tissues," Physics in Medicine and Biology, vol. 41, no. 11, pp. 2271-2293, 1996.

[31] N. Cho, J. Yoo, S.-J. Song, J. Lee, S. Jeon, and H.-J. Yoo, “The human body characteristics as a signal transmission medium for intrabody communication," IEEE Transactions on Microwave Theory and Techniques, vol. 55, no. 5, pp. 1080-1085, 2007.

[32] K. Hachisuka, T. Takeda, Y. Terauchi, K. Sasaki, H. Hosaka, and K. Itao, "Intra-body data transmission for the personal area network," Microsystem Technologies, vol. 11, no. 8-10, pp. 10201027, 2005.
[33] J. Mao, H. Yang, and B. Zhao, "An investigation on ground electrodes of capacitive coupling human body communication," IEEE Transactions on Biomedical Circuits and Systems, vol. 11, no. 4, pp. 910-919, 2017.

[34] J. Park, H. Garudadri, and P. P. Mercier, "Channel Modeling of Miniaturized Battery-Powered Capacitive Human Body Communication Systems," IEEE Transactions on Biomedical Engineering, vol. 64, no. 2, pp. 452-462, 2017.

[35] R. Xu, H. Zhu, and J. Yuan, "Electric-field intrabody communication channel modeling with finite-element method," IEEE Transactions on Biomedical Engineering, vol. 58, no. 3, pp. 705$712,2011$.

[36] J. Mao, H. Yang, Y. Lian, and B. Zhao, "A self-adaptive capacitive compensation technique for body channel communication," IEEE Transactions on Biomedical Circuits and Systems, vol. 11, no. 5, pp. 1001-1012, 2017.

[37] M. Amparo Callejón, D. Naranjo-Hernández, J. Reina-Tosina, and L. M. Roa, "Distributed circuit modeling of galvanic and capacitive coupling for intrabody communication," IEEE Transactions on Biomedical Engineering, vol. 59, no. 12, pp. 32633269, 2012.

[38] T. Handa, S. Shoji, S. Ike, S. Takeda, and T. Sekiguchi, "A very low-power consumption wireless ECG monitoring system using body as a signal transmission medium," in Proceedings of the International Solid State Sensors and Actuators Conference (Transducers '97), pp. 1003-1006, Chicago, Ill, USA.

[39] D. P. Lindsey, E. L. McKee, M. L. Hull, and S. M. Howell, "A new technique for transmission of signals from implantable transducers," IEEE Transactions on Biomedical Engineering, vol. 45, no. 5, pp. 614-619, 1998.

[40] K. Hachisuka, A. Nakata, T. Takeda et al., "Development and performance analysis of an intra-body communication device," in in 12th International Conference on Solid State Sensors, Actuators and Microsystems, vol. 105, pp. 109-115, Boston, Mass, USA, 2003.

[41] M. Wegmueller, Intra-body communication for biomedical sensor networks [Ph.D. thesis], Swiss Federal Institude of Technology Zurich, Swizerland, 2007.

[42] C. Chakraborty, B. Gupta, and S. K. Ghosh, "A review on telemedicine-based WBAN framework for patient monitoring," Telemedicine and e-Health, vol. 19, no. 8, pp. 619-626, 2013.

[43] M. Wegmueller, N. Felber, W. Fichtner et al., "Measurement System for the Characterization of the Human Body as a Communication Channel at Low Frequency," in Proceedings of the 2005 IEEE Engineering in Medicine and Biology 27th Annual Conference, pp. 3502-3505, Shanghai, China, 2005.

[44] K. Hachisuka, Y. Terauchi, Y. Kishi et al., "Simplified circuit modeling and fabrication of intrabody communication devices," Sensors and Actuators A: Physical, vol. 130-131, pp. 322330, 2006.

[45] B. Kibret, M. Seyedi, D. T. H. Lai, and M. Faulkner, "Investigation of galvanic-coupled intrabody communication using the human body circuit model," IEEE Journal of Biomedical and Health Informatics, vol. 18, no. 4, pp. 1196-1206, 2014.

[46] M. A. Callejon, L. M. Roa, J. Reina-Tosina, and D. NaranjoHernandez, "Study of Attenuation and Dispersion Through the Skin in Intrabody Communications Systems," IEEE Transactions on Information Technology in Biomedicine, vol. 16, no. 1, pp. 159-165, 2012.

[47] S. H. Pun, Y. M. Gao, P. Mak, M. I. Vai, and M. Du, "Quasi-static modeling of human limb for intra-body communications with 
experiments," IEEE Transactions on Information Technology in Biomedicine, vol. 15, no. 6, pp. 870-876, 2011.

[48] H. Schwan, "Electrical properties of tissues and cell suspensions: mechanisms and models," in Proceedings of the 16th Annual International Conference of the IEEE Engineering in Medicine and Biology Society, vol. 1, pp. A70-A71, Baltimore, Md, USA.

[49] K. Fujii and K. Ito, "Evaluation of the received signal level in relation to the size and carrier frequencies of the wearable device using human body as a transmission channel," in Proceedings of the IEEE Antennas and Propagation Society Symposium, 1, pp. 105-108, Monterey, Calif, USA, June 2004.

[50] K. Fujii, K. Ito, and S. Tajima, "A study on the receiving signal level in relation with the location of electrodes for wearable devices using human body as a transmission channel," in Proceedings of the 2003 IEEE International Symposium on Antennas and Propagation: URSI North American Radio Science Meeting, pp. 1071-1074, Columbus, Ohio, USA.

[51] K. Ito, M. Takahashi, and K. Fujii, “Transmission mechanism of wearable devices using the human body as a transmission channel," Artech House, pp. 65-92, 2006.

[52] J. Bae, H. Cho, K. Song, H. Lee, and H.-J. Yoo, "The signal transmission mechanism on the surface of human body for body channel communication," IEEE Transactions on Microwave Theory and Techniques, vol. 60, no. 3, pp. 582-593, 2012.

[53] S. Pun, Electromagnetic sub-MHz modeling of multilayer human limb for the galvanic coupling type intra-body communication [Ph.D. thesis], University of Macau, 2011.

[54] M. A. Callejon, D. Naranjo-Hernandez, J. Reina-Tosina, and L. M. Roa, "A comprehensive study into intrabody communication measurements," IEEE Transactions on Instrumentation and Measurement, vol. 62, no. 9, pp. 2446-2455, 2013.

[55] Z. D. Nie, J. J. Ma, Z. C. Li, H. Chen, and L. Wang, "Dynamic propagation channel characterization and modeling for human body communication," Sensors, vol. 12, no. 12, pp. 17569-17587, 2012.

[56] M. Seyedi and D. Lai, "Effect of limb joints and limb movement on intrabody communications for body area network applications," Journal of Medical and Biological Engineering, vol. 34, no. 3, pp. 276-283, 2014.

[57] X. M. Chen, S. H. Pun, J. F. Zhao, P. U. Mak, B. D. Liang, and M. I. Vai, "Effects of human limb gestures on galvanic coupling intrabody communication for advanced healthcare system," Biomedical Engineering Online, vol. 15, no. 1, article no. 60, 2016.

[58] X. M. Chen, P. U. Mak, S. H. Pun et al., "Improved transfer function with phase response from quasi-static field modeling for galvanic-type intra-body communication," in Proceedings of the 2013 IEEE 6th International Conference on Advanced Infocomm Technology, ICAIT 2013, pp. 101-103, twn, July 2013.

[59] X. M. Chen, P. U. Mak, S. H. Pun et al., "Study of channel characteristics for galvanic-type Intra-Body communication based on a transfer function from a quasi-static field model," Sensors, vol. 12, no. 12, pp. 16433-16450, 2012.

[60] Y.-T. Lin, Y.-S. Lin, C.-H. Chen, H.-C. Chen, Y.-C. Yang, and S.-S. Lu, "A 0.5-V biomedical system-on-a-chip for intrabody communication system," IEEE Transactions on Industrial Electronics, vol. 58, no. 2, pp. 690-699, 2011.

[61] M. S. Wegmueller, W. Fichtner, M. Oberle, and N. Kuster, "BPSK \& QPSK Modulated Data Communication for Biomedical Monitoring Sensor Network," in Proceedings of the Annual International Conference of the IEEE Engineering in Medicine and
Biology Society, pp. 2071-2074, New York, NY, USA, August 2006.

[62] L. Zhang, J. Huang, and L. Wang, "Experimental study on the optimum modulation schemes for human body channel communication," Bulletin of Advanced Technology Research, vol. 4, no. 9, pp. 29-33, 2010.

[63] X. M. Chen, P. U. Mak, S. H. Pun et al., "Initial investigation of channel capacity for galvanic coupling human body communication," in Proceedings of the 9th Biomedical Engineering International Conference, BMEiCON 2016, lao, December 2016.

[64] H. Zhu, R. Xu, and J. Yuan, "High speed intra-body communication for personal health care," in Proceedings of the Annual International Conference of the IEEE Engineering in Medicine and Biology Society (EMBC'09), pp. 709-712, IEEE, Minneapolis, Minn, USA, September 2009.

[65] J. A. Ruiz and S. Shimamoto, "Experimental evaluation of body channel response and digital modulation schemes for intrabody communications," in Proceedings of the IEEE International Conference on Communications (ICC '06), vol. 1, pp. 349-354, IEEE, Istanbul, Turkey, June 2006.

[66] E. Post, M. Reynolds, M. Gray, J. Paradiso, and N. Gershenfeld, "Intrabody buses for data and power," in Proceedings of the Digest of Papers First International Symposium on Wearable Computers, pp. 52-55, IEEE Computer Society, Cambridge, Mass, USA, 1997.

[67] N. Matsushita, S. Tajima, Y. Ayatsuka, and J. Rekimoto, "Wearable key: Device for personalizing nearby environment," in The Fourth International Symposium on Wearable Computers, pp. 119-126, IEEE, Atlanta, USA, 2000.

[68] K. Partridge, B. Dahlquist, A. Veiseh et al., "Empirical measurements of intrabody communication performance under varied physical configurations," in Symposiumon User Interface Software and Technology, pp. 183-190, ACM, Fla, USA, 2001.

[69] J. A. Ruiz and S. Shimamoto, "Statistical Modeling of Intra-body Propagation Channel," in Proceedings of the 2007 IEEE Wireless Communications and Networking Conference, pp. 2063-2068, Kowloon, China, March 2007.

[70] Z. Nie, F. Guan, J. Huang, and L. Wang, "Low power single-chip RF transceiver for human body communication," China Communications, vol. 9, no. 9, pp. 1-10, 2012.

[71] H. Cho, H. Lee, J. Bae, and H.-J. Yoo, “A 5.2?mw ieee 802.15.6 hbc standard compatible transceiver with power efficient delaylocked-loop based bpsk demodulator," in Solid-State Circuits Conference, pp. 297-300, IEEE, 2014.

[72] H. Cho, H. Kim, M. Kim, J. Jang, and H. Yoo, “79pJ/b 80Mb/s full-duplex transceiver and $42.5 \mathrm{jiW} 100 \mathrm{~kb} / \mathrm{s}$ super-regenerative transceiver for body channel communication," in Proceedings of the 2015 IEEE MTT-S 2015 International Microwave Workshop Series on RF and Wireless Technologies for Biomedical and Healthcare Applications (IMWS-BIO), pp. 14-15, Taipei, Taiwan, September 2015.

[73] S.-J. Song, N. Cho, S. Kim, J. Yoo, and H.-J. Yoo, "A 2Mb/s wideband pulse transceiver with direct-coupled interface for human body communications," in International Solid-State Circuits Conference, pp. 2278-2287, IEEE, San Francisco, Calif, USA, 2006.

[74] S.-J. Song, N. Cho, S. Kim, J. Yoo, S. Choi, and H.-J. Yoo, “A 0.9V $2.6 \mathrm{~mW}$ body-coupled scalable PHY transceiver for body sensor applications," in Proceedings of the 54th IEEE International Solid-State Circuits Conference, ISSCC 2007, pp. 355-609, usa, February 2007. 
[75] N. Cho, L. Yan, J. Bae, and H.-J. Yoo, "A 60 kb/s10 Mb/s adaptive frequency hopping transceiver for interference-resilient body channel communication," IEEE Journal of Solid-State Circuits, vol. 44, no. 3, pp. 708-717, 2009.

[76] K. Katsu, D. Anzai, and J. Wang, "Performance evaluation on correlation detection and energy detection for ultra widebandimpulse radio communication with multi-pulse position modulation scheme in implant body area networks," IET Communications, vol. 7, no. 13, pp. 1430-1436, 2013.

[77] J. Huang, L. Wang, D. Zhang, and Y. Zhang, "A low-frequency low-noise transceiver for human body channel communication," in Proceedings of the 2009 IEEE Biomedical Circuits and Systems Conference (BioCAS), pp. 37-40, Beijing, China, November 2009.

[78] C.-H. Hyoung, S.-W. Kang, S.-O. Park, and Y.-T. Kim, “Transceiver for human body communication using frequency selective digital transmission," ETRI Journal, vol. 34, no. 2, pp. 216$225,2012$.

[79] C. Chung, C. Lin, and J. Yang, "Time-domain characteristics of body channel communication (BCC) and BCC transceiver design," in Proceedings of the 2016 International Symposium on VLSI Design, Automation and Test (VLSI-DAT), pp. 1-4, Hsinchu, Taiwan, April 2016.

[80] M. Shinagawa, M. Fukumoto, K. Ochiai, and H. Kyuragi, "A near-field-sensing transceiver for intrabody communication based on the electrooptic effect," IEEE Transactions on Instrumentation and Measurement, vol. 53, no. 6, pp. 1533-1538, 2004.

[81] H.-J. Yoo, S.-J. Song, N. Cho, and H.-J. Kim, in Proceedings of the 4th International Workshop on Wearable and Implantable Body Sensor Networks (BSN), pp. 15-20, Springer, 2007.

[82] C. O. Asogwa, S. F. Collins, P. McLaughlin, and D. T. H. Lai, "A galvanic coupling method for assessing hydration rates," Electronics (Switzerland), vol. 5, article 39, no. 3, 2016.

[83] X. M. Chen, S. Barma, S. H. Pun, M. I. Vai, and P. U. Mak, "Direct Measurement of Elbow Joint Angle Using Galvanic Couple System," IEEE Transactions on Instrumentation and Measurement, vol. 66, no. 4, pp. 757-766, 2017.

[84] "IEEE standard for safety levels with respect to human exposure to the radio frequency electromagnetic fields, $3 \mathrm{khz}$ to $300 \mathrm{ghz}$," IEEE Std C95.1-2005, 2005.

[85] A. Ahlbom, U. Bergqvist, J. Bernhardt et al., "Guidelines for limiting exposure to time-varying electric, magnetic, and electromagnetic fields (up to $300 \mathrm{GHz}$ ). International commission on non-ionizing radiation protection," Health Physics, vol. 74, no. 4, pp. 494-522, 1998.

[86] M. S. Wegmueller, M. Oberle, N. Felber, N. Kuster, and W. Fichtner, "Signal transmission by galvanic coupling through the human body," IEEE Transactions on Instrumentation and Measurement, vol. 59, no. 4, pp. 963-969, 2010.

[87] M. Neuman, Biopotential Electrodes, John Wiley and Sons, NJ, USA, 1998.

[88] D. R. Merrill, M. Bikson, and J. G. R. Jefferys, "Electrical stimulation of excitable tissue: Design of efficacious and safe protocols," Journal of Neuroscience Methods, vol. 141, no. 2, pp. 171198, 2005.

[89] J. Sakai, L.-S. Wu, H.-C. Sun, and Y.-X. Guo, "Baluns effect on the measurement of transmission characteristics for intrabody communication channel," in IEEE MTT-S International Microwave Workshop on RF and Wireless Technologies for Biomedical and Healthcare Applications (IMWS-BIO), pp. 1-3, 2013.
[90] M. A. Callejon, J. Reina-Tosina, D. Naranjo-Hernandez, and L. M. Roa, "Measurement issues in galvanic intrabody communication: Influence of experimental setup," IEEE Transactions on Biomedical Engineering, vol. 62, no. 11, pp. 2724-2732, 2015.

[91] Ž. L. Vasić, I. Krois, and M. Cifrek, "Effect of transformer symmetry on intrabody communication channel measurements using grounded instruments," Automatika - Journal for Control, Measurement, Electronics, Computing and Communications, vol. 57, no. 1, pp. 15-26, 2016.

[92] J. W. Li, X. M. Chen, S. H. Pun et al., "Bit error rate estimation for galvanic-type intra-body communication using experimental eye-diagram and jitter characteristics," in Proceedings of the 2013 35th Annual International Conference of the IEEE Engineering in Medicine and Biology Society, EMBC 2013, pp. 5195-5198, jpn, July 2013.

[93] IEEE, Standard for Local and metropolitan area networks, Std., 2011.

[94] B. S. I. G. SIG, "Adopted bluetooth core specification (core version 4.0)," 2010, https://www.bluetooth.com/specifications/ adopted-specifications.

[95] S. Mandal and R. Sarpeshkar, "Power-efficient impedance-modulation wireless data links for biomedical implants," IEEE Transactions on Biomedical Circuits and Systems, vol. 2, no. 4, pp. 301-315, 2008.

[96] Ž. Lučev, I. Krois, and M. Cifrek, "Effect of body positions and movements in a capacitive intrabody communication channel from $100 \mathrm{kHz}$ to $100 \mathrm{MHz}$," in Proceedings of the 2012 IEEE International Instrumentation and Measurement Technology Conference, I2MTC 2012, pp. 2791-2795, aut, May 2012. 


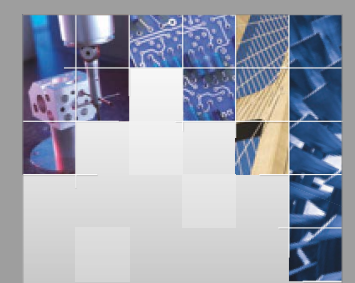

\section{Enfincering}
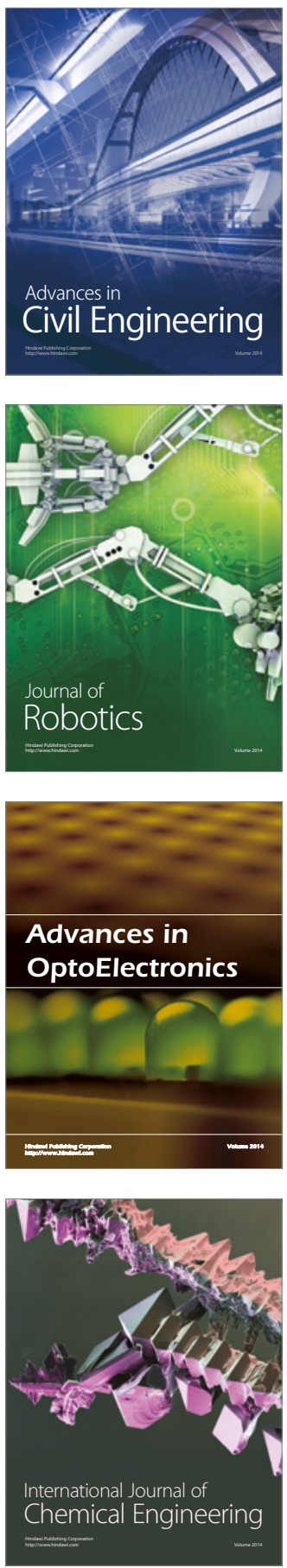

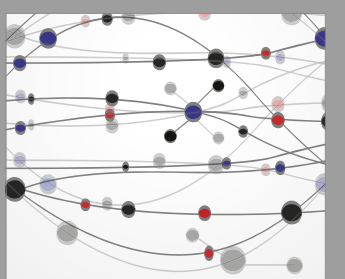

The Scientific World Journal

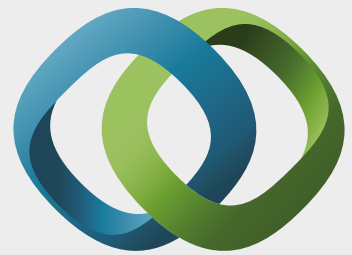

\section{Hindawi}

Submit your manuscripts at

https://www.hindawi.com
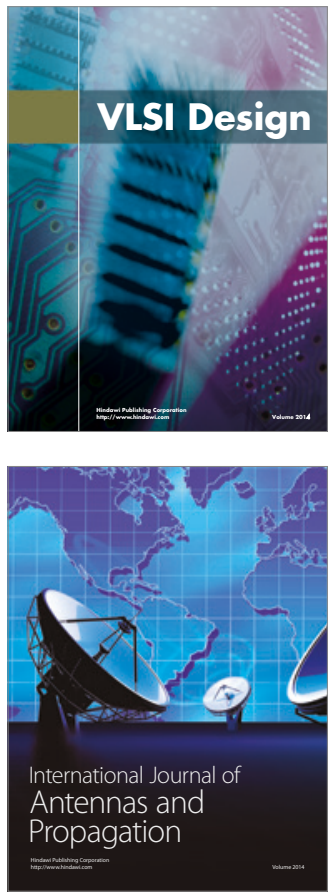

\section{Rotating}

Machinery
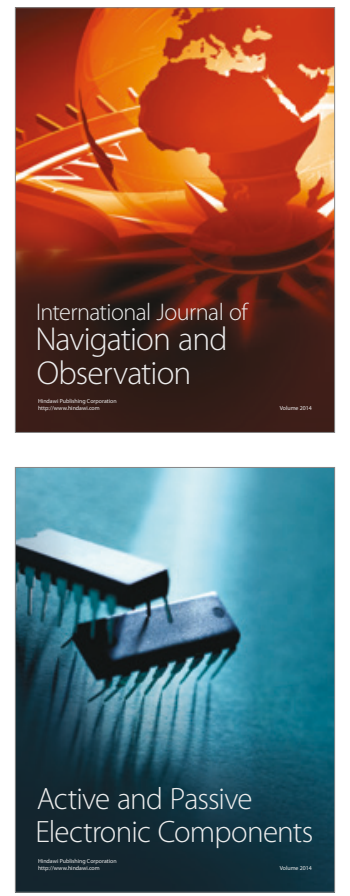
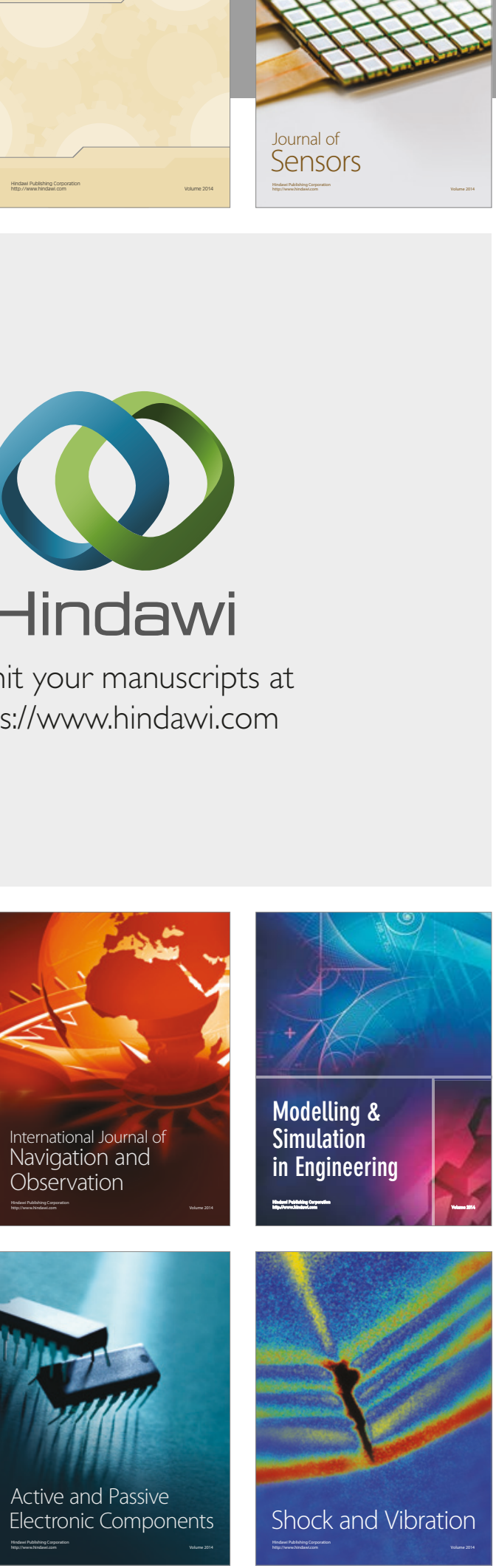
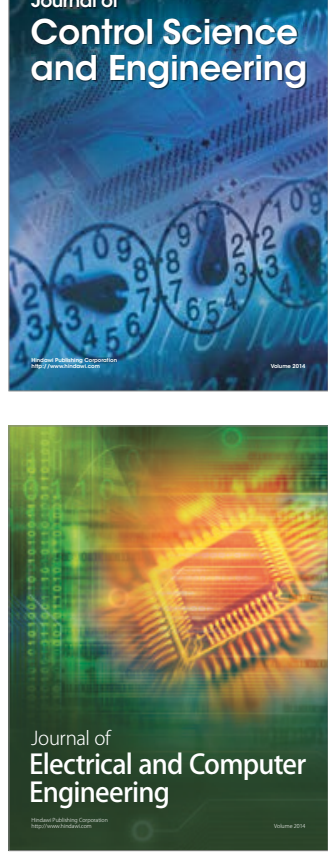

Distributed

Journal of

Control Science

and Engineering
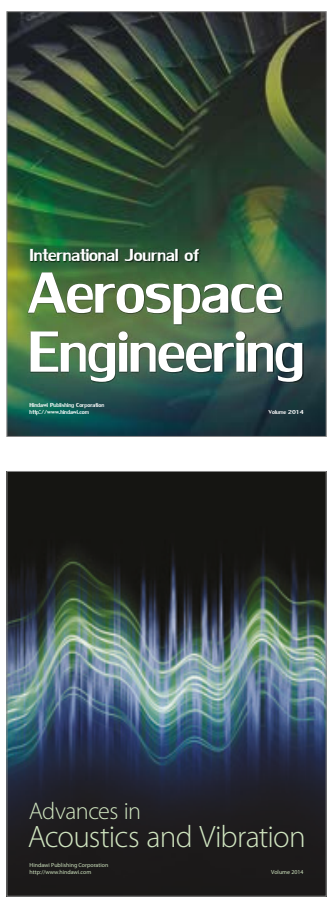

Sensor Networks 